Research articles

\title{
Magnetooptical response to tunnel magnetoresistance in manganite films with a variant structure
}

\author{
A.V. Telegin ${ }^{\text {a,*, }}$, S. Barsaume ${ }^{b}$, V.A. Bessonova ${ }^{a}$, Yu.P. Sukhorukov ${ }^{a}$, A.P. Nosov ${ }^{a}$, A.V. Kimel' ${ }^{\text {, }}$, \\ E.A. Gan'shina ${ }^{c}$, A.N. Yurasov ${ }^{\mathrm{d}}$, E.A. Lysina ${ }^{\mathrm{d}}$ \\ ${ }^{a}$ M.N. Miheev Institute of Metal Physics Ural Division of RAS, 620137 Yekaterinburg, Russia \\ ${ }^{\mathrm{b}}$ Radboud University, Institute of Molecules and Materials, 6525 ED Nijmegen, The Netherlands \\ ${ }^{\mathrm{c}}$ Lomonosov Moscow State University (MSU), 119991 Moscow, Russia \\ ${ }^{\mathrm{d}}$ Moscow Technological University (MIREA), 119454 Moscow, Russia
}

\section{A R T I C L E I N F O}

\section{Article history:}

Received 10 July 2017

Received in revised form 22 September

2017

Accepted 2 October 2017

Available online 15 October 2017

\section{Keywords:}

Manganite films

Variant structure

Grain boundaries

Colossal and tunnel magnetoresistance

Magnetotransmission

Infrared

\begin{abstract}
A B S T R A C T
The optical, magnetooptical, electrical, and magnetic properties of $\mathrm{La}_{0.67} \mathrm{Ba}_{0.33} \mathrm{MnO}_{3}$ films grown on the $\mathrm{SrTiO}_{3}$ and $\mathrm{ZrO}_{2}\left(\mathrm{Y}_{2} \mathrm{O}_{3}\right)$ substrates have been studied. The temperature and magnetic field dependences of magnetotransmission of light in the $\mathrm{La}_{0.67} \mathrm{Ba}_{0.33} \mathrm{MnO}_{3} / \mathrm{ZrO}_{2}\left(\mathrm{Y}_{2} \mathrm{O}_{3}\right)$ film with a variant structure exhibit features which can be attributed to an optical response to tunnel magnetoresistance - tunnel magnetotransmission. Unlike the optical response to colossal magnetoresistance, existing near the Curie temperature only, the tunnel magnetotransmission increases at $T \rightarrow 0 \mathrm{~K}$, has a complex hysteresis behavior with the change of sign of the effect, and saturates in magnetic fields above 1 kOe. The observed tunnel magnetotransmission has been interpreted within the magnetorefractive effect theory developed for granular films.
\end{abstract}

(c) 2017 Elsevier B.V. All rights reserved.

\section{Introduction}

Numerous works have been devoted to identification of the physical mechanisms of optical response to colossal magnetoresistance (CMR) in doped manganites (see, for example, [1,2] and references therein). Such response can be considered as magnetoreflection and magnetotransmission effects which take place in the CMR manganites in the infrared spectral range near the Curie temperature $\left(T_{C}\right)$. Apart from CMR in granular manganite-based systems there exists the specific lowtemperature magnetoresistance (MR) which is due to scattering and tunneling of spin- polarized charge carriers thorough grain boundaries - the so-called tunnel magnetoresistance (TMR) [3]. Noticeable TMR values were observed in manganite films with an equivalent (variant) structure [4]. Such structure can be formed

\footnotetext{
Abbreviations: CMR, colossal magnetoresistance; MR, magnetoresistance; TMR tunnel magnetoresistance; IR, infrared; LBMO, $\mathrm{La}_{0.67} \mathrm{Ba}_{0.33} \mathrm{MnO}_{3} ; \mathrm{SrTiO}_{3}, \mathrm{STO} ; \mathrm{YSZ}$, $\mathrm{ZrO}_{2}\left(\mathrm{Y}_{2} \mathrm{O}_{3}\right)$.

* Corresponding author at: 620137 S. Kovalevskaya Str., 18, IMP, Yekaterinburg, Russia.

E-mail address: telegin@imp.uran.ru (A.V. Telegin).
}

during growth of manganite films on the $\mathrm{ZrO}_{2}\left(\mathrm{Y}_{2} \mathrm{O}_{3}\right)$ single crystalline substrates and can be considered as consisting of a set of identical crystallites - structural domains of equal size with fixed number and concentration of coherent high-angular boundaries within the film volume. This is the main structural difference from polycrystalline films. The variant structure leads to formation of highly conductive ferromagnetic nanosized grains (crystallites) separated by lowly conductive grain boundaries. As a result, an additional contribution to MR associated with TMR appears [47]. Taking into account different temperature dependences of $\operatorname{CMR}(T)$ and $\operatorname{TMR}(T)$ it is easy to separate their contributions to the total $\operatorname{MR}(T)$ dependence by choosing the manganite composition with high $T_{C}$, for example, the $\mathrm{La}_{2 / 3} \mathrm{Ba}_{1 / 3} \mathrm{MnO}_{3}$, and wide temperature range of interest. Though the TMR in manganites is wellknown, however, there is clear lack of evidences for optical response to tunnel magnetoresistance. Earlier the optical response to TMR was considered theoretically for granular allows [8] and metallic superlattices $[9,10]$. As far as we know the few experimental attempts $[11,12]$ to reveal an optical response to TMR in manganites have not been successful. The main problem of these attempts is in the absence of low-temperature optical measurements (close to zero Kelvin) and high absorbance of manganites 
in a ferromagnetic state. In this work, we have made a comparative analysis of experimental data on optical properties and electrical resistance for the thin $\mathrm{La}_{0.67} \mathrm{Ba}_{0.33} \mathrm{MnO}_{3}$ films in the presence and absence of the variant structure in the wide temperature range. As a result, the contribution of optical response to TMR in magnetotransmission of unpolarized light was revealed.

\section{Materials and methods}

Epitaxial films of the $\mathrm{La}_{0.67} \mathrm{Ba}_{0.33} \mathrm{MnO}_{3}$ composition with the thickness of $d=80 \mathrm{~nm}$ were grown on the single-crystal $\mathrm{SrTiO}_{3}$ ( $\left.\begin{array}{lll}0 & 0 & 1\end{array}\right)$ (the cubic lattice constant $a_{0}=3.905 \AA$ ), hereafter denoted as LBMO/STO, and the $\mathrm{ZrO}_{2}\left(\mathrm{Y}_{2} \mathrm{O}_{3}\right)\left(\begin{array}{lll}1 & 0 & 0\end{array}\right)$ (the cubic lattice constant $\left.a_{0}=5.140 \AA\right)$, hereafter denoted as LBMO/YSZ, substrates. The square-shaped substrates with the surface area of $1 \mathrm{~cm}^{2}$ were used. The growth process was carried out with the substrate temperature of $T_{S}=1000 \mathrm{~K}$ and the oxygen pressure of $P=0.4 \mathrm{mbar}$ by pulsed laser deposition using the excimer KrF laser with $\lambda=247$ $\mathrm{nm}$. The films were postannealed in the deposition chamber under oxygen flow at atmospheric pressure and $T=730 \mathrm{~K}$ to improve the oxygen stoichiometry. The elemental composition of the films was determined by EDAX analysis using the Inspect $\mathrm{F}$ (FEI) scanning electron microscope. According to X-ray diffraction data, the films were single-phase, highly oriented, and had an orthorhombic perovskite structure. The effective Curie temperature $\left(T_{C}^{*}\right)$ of the films was determined from magneto-optical and magnetization measurements using the Lake Shore 7400 VSM vibration sample magnetometer. The $T_{C}^{*}$ value of $\approx 290 \mathrm{~K}$ was found for the LBMO/YSZ film while $T_{C}^{*} \approx 300 \mathrm{~K}$ was determined for the LBMO/STO film. Optical properties of the films were studied using the home made IR prism spectrometer in IMP UB RAS and the Vortex 80v Fourierspectrometer in the Radboud University. Measurements were carried out for unpolarized light in the infrared spectral range $1 \leq \lambda \leq$ $22 \mu \mathrm{m}$, temperature interval from 10 to $350 \mathrm{~K}$, and magnetic fields of up to $H=7.5$ kOe oriented along (the Voigt geometry, $H \perp q$ ) and perpendicular to (the Faraday geometry, $\mathrm{H} \| \mathrm{q}$ ) the surface of the film. The films were arbitrary oriented during these experiments. The coefficient of absorption of light was calculated as $K=(1 / d) \ln$ $\left[(1-R)^{2} / t\right]$, where $t$ is the transmission of light and $R$ is the coefficient of reflection. The magnetotransmission was defined as $\Delta t / t=$ $\left[t_{H}-t_{0}\right] / t_{0}$, where $t_{H}$ and $t_{0}$ are the intensities of the transmitted light in the presence and absence of a magnetic field $H$. The relative error in determination of the magnetotransmission was about $0.1 \%$. Electro- and magnetoresistance of the films $\Delta \rho / \rho_{0}=\left[\rho_{H}-\right.$ $\left.\rho_{0}\right] / \rho_{0}$ were measured by the two-point contact method with ultrasonically soldered silver contacts in magnetic fields of up to 8 kOe.

\section{Results and discussion}

\subsection{Spectral dependences}

The shape of spectral dependences of the absorption coefficient $K$ for the films (inset in Fig. 1b) is similar to those observed for the single crystals $[13,14]$ and manganite films with CMR (see, for example [15]). The strong absorption at short wavelengths is attributed to onset of the $d-d$ transitions $\left({ }^{5} E_{g}-{ }^{5} T_{2 g}\right)$ in $\mathrm{Mn}^{3+}$ ions forming the absorption edge in manganite films $[16,17]$. The spectral dependence of $K$ follows the $K(h \omega)=\left(h \omega-\varepsilon_{g}\right)^{3 / 2}$ law with the optical gap $\varepsilon_{g}=0.4 \mathrm{eV}$. Consequently, the absorption edge is formed by the direct forbidden interband transitions [15]. Upon cooling below $T_{C}$ the absorption, as well as reflection, of the film increase due to the strong contribution from free charge carriers in the mid-IR range (inset in Fig. 1b) [1,12]. Application of mag- netic field results in increase of the absorption too, thus resulting in negative $\Delta t / t$.

The spectra of magnetotransmission of light for the LBMO/YSZ and LBMO/STO films recorded at $T=295 \mathrm{~K}$ are shown in Fig. $1 \mathrm{a}$. The magnitude of $\Delta t / t$ for LBMO/YSZ is two times lower than that for LBMO/STO which can be due only to the presence of structural domains in the LBMO/YSZ film. Variation from in-plane $H \perp q$ geometry of the experiment to the out-of-plane $H \| q$ one does not principally affect the magnitude of $\Delta t / t$ (not shown) due to the maximal fluctuations of the magnetic moments near $T_{\mathrm{C}}$. In general, the $\Delta t / t$ spectra are formed by competition of a positive contribution from the interband transitions at $\lambda<1.5 \mu \mathrm{m}$ and a negative contribution associated with the interaction of light with delocalized charge carriers and localized states at $\lambda>1.5 \mu \mathrm{m}$. According to the theory of the magnetorefractive effect developed for manganites with CMR [see, for example, 1 and references therein] $\Delta t$ ) $t$ should reach a maximum at $\lambda \sim 20 \mu \mathrm{m}$ in agreement with the $\omega \tau \sim 1$ condition, where $\tau$ is the relaxation time of the electron momentum $\left(1 / \tau=2.3 \times 10^{14} \mathrm{~s}^{-1}[14]\right)$. This explains the observed increase of $\Delta t / t$ at $\lambda>10 \mu \mathrm{m}$. This theory is applicable only for optimally doped manganites [12] in the spectral range of interaction of light with delocalized charge carriers (the near and middle IR spectral ranges). According to (1) the estimated values of $\Delta t / t$ for the films under study are in good agreement with the experimental data (see Fig. 1a).

$\Delta t / t=0.5 \cdot \Delta \rho / \rho(0) \cdot t(0) \cdot k^{2} \cdot\left(2 n^{2}+n\right) /\left(n^{2}+k^{2}\right)$

where the indexes of refraction $n$ and $k$ were subtracted from the absorption and reflection data (not shown).

Small differences between the experimental and calculated data are probably due to underestimation of the contribution of localized states and interband transitions in the theoretical model [1]. The most important result obtained is in revealing the noticeable magnetotransmission effect for the films with the variant structure far below $T_{C}$ (at $T<200 \mathrm{~K}$ ) where the TMR contribution exists even in the low field of $H=2 \mathrm{kOe}$. Upon $T \rightarrow 0 \mathrm{~K}$ magnetotransmission increases and reaches a magnitude close to that observed near $T_{C}$. In other words, we can observe both a pronounced optical response to the CMR near $T_{C}$ and optical response to TMR at low temperatures - the so-called tunnel magnetotransmission. As one can see, the $\Delta t / t$ spectra at low temperatures are identical to those at $T \sim T_{C}$ (Fig. 1b). The more detailed information can be derived from comparative analysis of the temperature and magnetic field dependencies of $\Delta t / t$ and MR effects.

In order to separate the contributions related to optical response to CMR and TMR in the manganite film we tried to model the $\Delta t / t(\lambda, T)$ dependence at different temperatures starting from $13 \mathrm{~K}$ (formulae 2). The same approach was used for calculation of magnetorefractive effect in granular systems with TMR [18].

$\Delta t / t\left(T_{2}\right)=\left[\Delta t / t\left(T_{1}\right) \times T_{2} \times \Delta \rho / \rho\left(T_{1}\right)\right] / T_{1} \lambda \alpha \Delta \rho / \rho\left(T_{2}\right)$

where $T_{1}=13 \mathrm{~K}, T_{2}=80 \mathrm{~K}, 200 \mathrm{~K}$ and $300 \mathrm{~K}, \Delta t / t$ and $\Delta \rho / \rho$ are magnetotransmission and MR of the film with the variant structure recorded at the appropriate temperatures.

One can see that this modeling gives a quite good qualitative result for $\Delta t / t$ at low temperatures and underestimated magnitude of the effect at temperatures close to $T_{C}(300 \mathrm{~K}$ ) (Fig. 1b). This can be attributed to two mechanisms of $\Delta t / t$. The first one is related to the existence of ferromagnetic domains with high conductivity, the $\Delta \rho / \rho(T)$ dependence having maximum near $T_{C}$, and comparable in magnitude with magnetotransmission for the film without variant structure. The second one is related to scattering at the grain boundaries of crystallographic structural grains with lowconductivity. At the temperature scale a borderline between these contributions is located near $T=200 \mathrm{~K}$, where for the LBMO/STO 


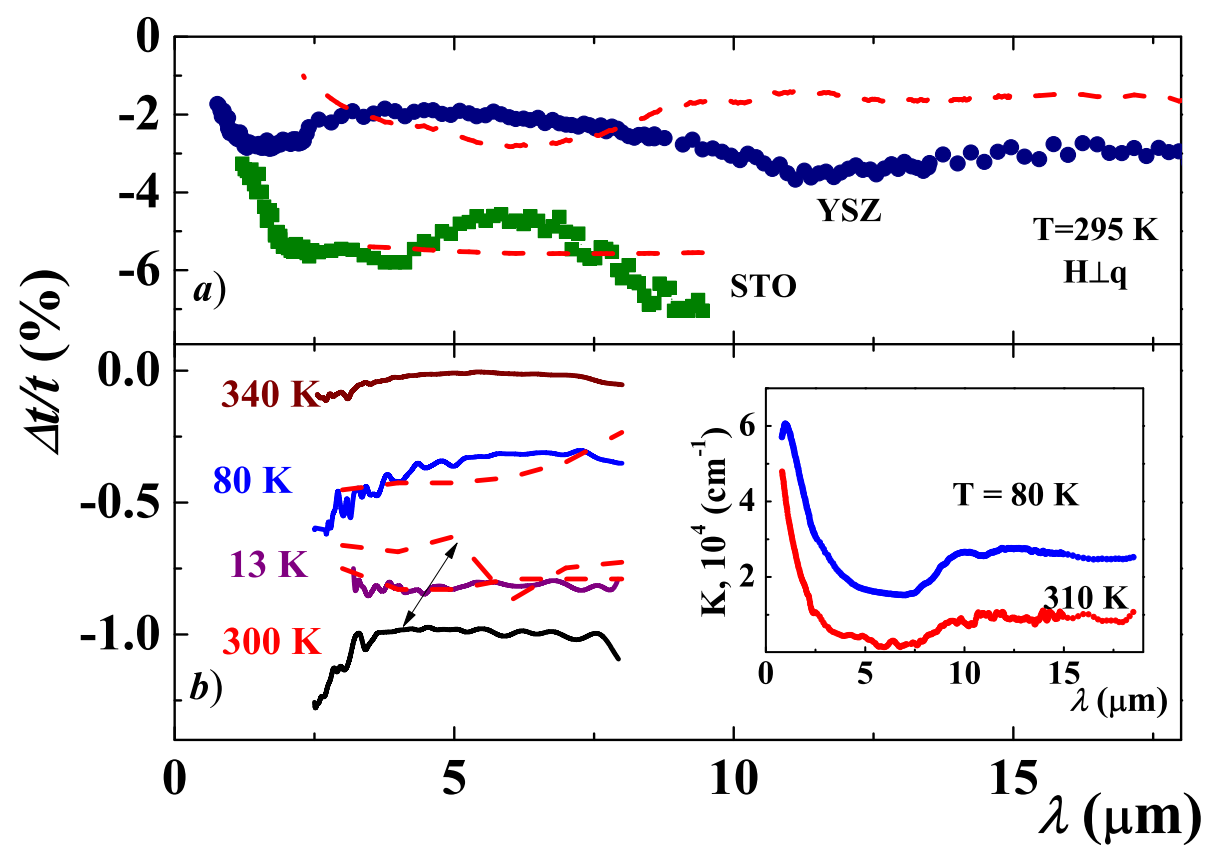

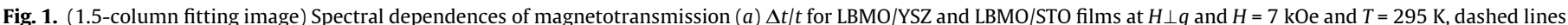

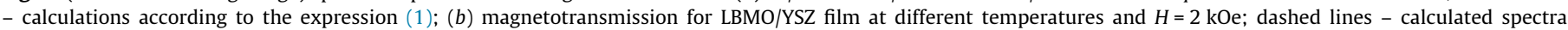
according to the expression (2). In the inset: absorption spectra for the LBMO/YSZ film.

film $\Delta t / t$ is vanished. Possible size-dependent effect in the film with nanosized structural grains and magnetic domains should also be taken into account. Thus our modeling can be applied for estimation of the optical responses to TMR and CMR for such complex materials as manganites.

\subsection{Temperature dependences}

The temperature and magnetic field dependencies of $\Delta t / t$ as well as of $\Delta \rho / \rho_{0}$ differed significantly for the LBMO/YSZ and

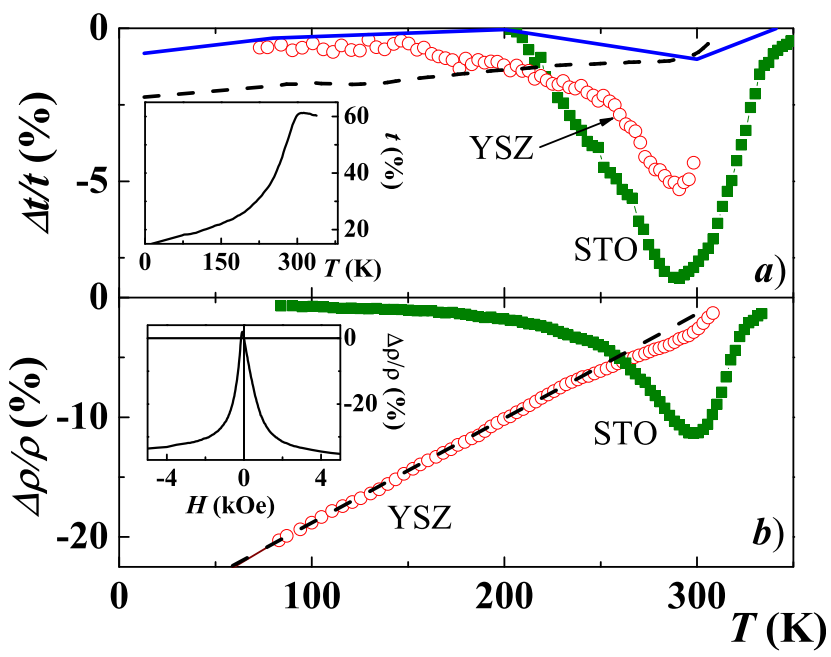

Fig. 2. (1.5-column fitting image) Temperature dependences of (a) magnetotransmission $\Delta t / t$ and (b) magnetoresistance $\Delta \rho / \rho$ for LBMO films on different substrates at $H=7.5 \mathrm{kOe}$. Solid line $-\Delta t / t(T)$ dependence obtained for the LBMO/ YSZ film at $H=2 \mathrm{kOe}$; dashed lines - the results of calculations of $\Delta t / t$ and $\Delta \rho / \rho$ according to the expression (1) and $f=a+b /(T+c)$ [3], respectively. In the inset: $(a)$ the temperature dependence of the transparency $t(T)$ at $\lambda=6 \mu \mathrm{m}$ and $(b)$ the field dependence of $\Delta \rho / \rho(H)$ at $T=4.2 \mathrm{~K}$ for the LBMO/YSZ film.
LBMO/STO films. The $\Delta t / t(T)$ and $\Delta \rho / \rho(T)$ temperature dependences for the LBMO/STO film have a clear maxima near $T_{C}$ (Fig. 2a) associated with the suppression fluctuations of the magnetic moments by magnetic field near $T_{C}$ (Fig. 2b). In the case of the LBMO/YSZ film the $\Delta \rho / \rho(T)$ dependence shows only a shoulder near $T_{C}$ but $\Delta t / t(T)$ dependence has a maximum of $\sim-5.5 \%(H=$ $7.5 \mathrm{kOe}$ ) (Fig. 2a). This maximum can be reasonably related to the optical response to CMR. The values of $\Delta t / t$ for the LBMO/ STO and LBMO/YSZ films are almost the same near $T_{C}$. Unlike the LBMO/STO film the $\mathrm{La}_{0.67} \mathrm{Ba}_{0.33} \mathrm{MnO}_{3} / \mathrm{ZrO}_{2}\left(\mathrm{Y}_{2} \mathrm{O}_{3}\right)$ one with the variant structure demonstrates the significant low-temperature magnetoresistance (up to $30 \%$ at $T \rightarrow 0 \mathrm{~K}$ ) associated with the tunneling of spin-polarized electrons through the structural grain boundaries. Similar $\Delta \rho / \rho(T)$ behavior was observed for other manganite films with variant structure [5,9]. Moreover, we revealed substantial magnetotransmission in the LBMO/YSZ film at $T<$ $150 \mathrm{~K}$ (Fig. 2a). The value of $\Delta t / t(T)$ reaches about $-1 \%$ at $T=13 \mathrm{~K}$ which is comparable with the value of $\Delta t / t$ near $T_{C}$.

In nanomaterials TMR is usually described by the expression $\Delta \rho / \rho \approx-P^{2} m^{2}(1+\sqrt{ }(C / T))$, where $C$ is a constant $[3,19]$. In our case the experimental curve $\Delta \rho / \rho(T)$ is better described by the $f=a+b /$ $(T+c)$ dependence proposed for bulk polycrystalline manganites (Fig. 2b) [20]. The authors of $[21,22]$ experimentally showed that such temperature dependence of TMR in manganite films on YSZ substrates is associated with destruction of the coherence of the structural domain boundaries and appearance of polycrystallinity. Estimation of the degree of spin-polarization at $T \rightarrow 0 \mathrm{~K}$ using the expression $\Delta \rho / \rho=2 P^{2} /\left(1-P^{2}\right)$ from [3] gives the value of $P \approx$ 0.36 which is close to $P \approx 0.41$ obtained for the $\operatorname{La}_{0.8} \mathrm{Ag}_{0.1} \mathrm{MnO}_{3+\delta} /$ YSZ film [11]. Using the expression (1) and the experimental temperature dependence of TMR we calculated the $\Delta t / t(T)$ dependence. Good correlation between calculated and experimental data was obtained only at $T=13 \mathrm{~K}$ (taking into account the value of $H$ ). Above $150 \mathrm{~K}$ one should consider an extra significant contribution related to the optical response to the metal-insulator transition (inset in Fig. 2a) within structural domain that has a maximum near $T_{C}$ for the film material. 


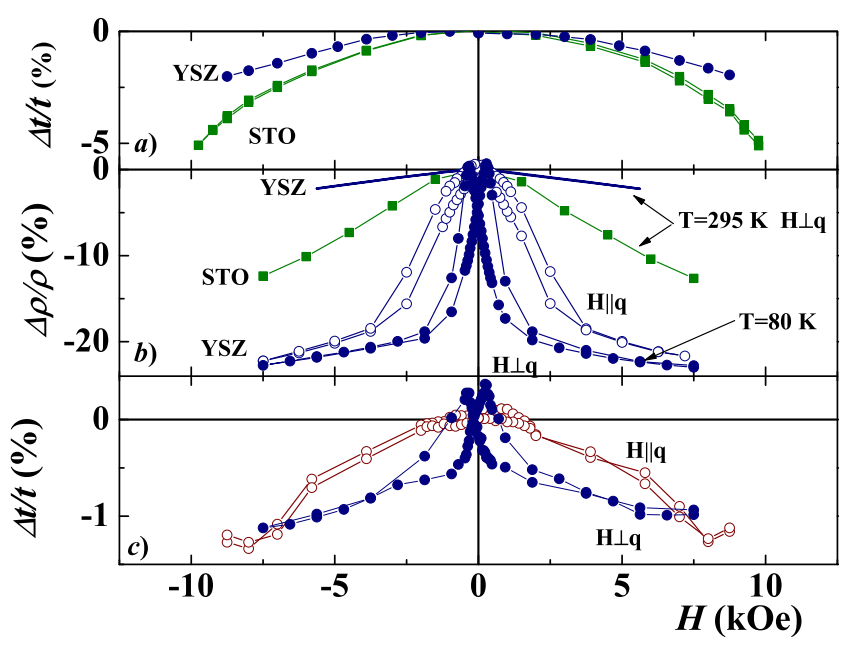

Fig. 3. (1.5-column fitting image) Magnetic field dependences of (a) magnetotransmission $\Delta t / t$ for the LBMO/YSZ and LBMO/STO films at $\lambda=6 \mu \mathrm{m}, T=295 \mathrm{~K}$ and $H \perp q$; (b) magnetoresistance $\Delta \rho / \rho$ for the LBMO/ZYO and LBMO/STO films at different temperatures and magnetic field direction; $(c)$ magnetotransmission $\Delta t / t$ for the LBMO/YSZ film at $\lambda=6 \mu \mathrm{m}$ and $T=80 \mathrm{~K}$.

\subsection{Magnetic field dependences}

The differences between the CMR and TMR, as well as optical responses to CMR and TMR, are most clearly manifest themselves in the magnetic field dependences of these effects (Fig. 3b). First of all, upon variation of the geometry of experiment and application of magnetic field either along $(H \| q)$ or perpendicular $(H \perp q)$ to the film's surface, the influence of the form factor was 3 times less than the expected $N=4 \pi$ for thin films. The reduced $N$ value for the film with variant structure may be explained by the influence of structural domain boundaries on the dynamics of magnetic domains in applied magnetic field [21].

In the vicinity of the Curie temperature the magnetic field dependences of $\Delta t / t(H)$ and $\Delta \rho / \rho(H)$ for both films are similar and typical for manganites with CMR $[1,2]$. The magnetic field dependences of colossal magnetoresistance and magnetotransmission are even on magnetization and demonstrate the absence of saturation in magnetic fields of up to $8 \mathrm{kOe}$ regardless the direction of the in-plane field relative to the film surface. They are associated with the maximal fluctuations of the magnetic moments near $T_{C}$. At low temperatures $(T<150 \mathrm{~K})$ the CMR contribution in thin films becomes zero, while TMR appeared only in the LBMO/YSZ film (inset in Fig. 2b).

In contrast to CMR, the tunnel magnetoresistance has a complex hysteresis behavior with a positive value of up to $\sim+0.7 \%$ in the area of technical magnetization associated with reorientation of magnetic domains in the fields below $H_{1}=300 \mathrm{Oe}$, and a negative value of up to $\sim-20 \%$ associated with the tunneling effect at $\mathrm{H}_{2}$ $=1.9 \mathrm{kOe}(H \perp q)$ and $H^{*}{ }_{2}=3.8 \mathrm{kOe}(H \| q)$ (Fig. 3b). Relatively high value of $\mathrm{H}_{2}$ is due to the planar anisotropy of the film with TMR $[7,22]$. As can be seen from the Fig. 3c, magnetotransmission strictly follows the TMR field dependences and reaches about $-1.1 \%$ at $H=7.5 \mathrm{kOe}$. It differs significantly from the behavior of the magnetotransmission near $T_{C}$ and is related to the optical response to TMR - the tunnel magnetotransmission. Weak hysteresis of tunnel magnetotransmission is preserved even in case of the Faraday geometry with $H \| q$. One can notice the different values of form-factor $N$ obtained from the $\Delta t / t(H)$ and $M / M_{s}(H)$ dependences (not shown). Under these circumstances, additional study of the anisotropy of TMR and related to it tunnel magnetotransmission in the film with a variant structure for in-plane oriented magnetic field can be suggested.

\section{Conclusion}

Optical, magnetooptical, electrical, and magnetic properties of the $\mathrm{La}_{0.67} \mathrm{Ba}_{0.33} \mathrm{MnO}_{3}$ magnetoresistive epitaxial films grown on the $\mathrm{SrTiO}_{3}$ and $\mathrm{ZrO}_{2}\left(\mathrm{Y}_{2} \mathrm{O}_{3}\right)$ substrates have been studied. Apart from colossal magnetoresistance, the films on both substrates show significant magnetotransmission (5\%) of unpolarized light in the wide infrared range with a maximum near the Curie temperature (290-300 K). The $\mathrm{La}_{0.67} \mathrm{Ba}_{0.33} \mathrm{MnO}_{3} / \mathrm{ZrO}_{2}\left(\mathrm{Y}_{2} \mathrm{O}_{3}\right)$ film with the variant structure demonstrates the low-temperature magnetoresistance (up to $30 \%$ at $T=4.2 \mathrm{~K}$ ) associated with the tunneling of spin-polarized carriers through the boundaries of structural domains. Temperature dependence of this effect was well approximated by the function $f=a+b /(T+c)$, which points to polycrystalline structure of the film. The degree of spin-polarization of charge carriers was estimated as $P \approx 0.36$.

It is worth to notice, a direct optical response to the tunnel magnetoresistance was revealed in magnetotransmission of light for the $\mathrm{La}_{0.67} \mathrm{Ba}_{0.33} \mathrm{MnO}_{3} / \mathrm{ZrO}_{2}\left(\mathrm{Y}_{2} \mathrm{O}_{3}\right)$ film for the first time - tunnel magnetotransmission. The so-called tunnel magnetotransmission follows the temperature and field dependences of tunnel magnetoresistance and reach the value of $-1.1 \%$ at $T=13 \mathrm{~K}$ and $H=$ $2 \mathrm{kOe}$. The tunnel magnetotransmission depends on the direction of the magnetic field along or perpendicular to the film plane due to the contribution from the magnetic domain dynamics and the demagnetizing factor. Calculations of the spectral and temperature dependences of magnetotransmission in the framework of the magnetorefractive effect theory developed earlier for granular systems show good correlation with the experimental data.

The appearance of a substantial magnetoresistance and magnetotransmission in manganite films in relatively weak magnetic fields is of considerable interest for potential fabrication of optoelectronic sensors of magnetic field with high-sensitivity. For these purposes one can use both the appearance of sharp maxima of positive magnetoresistance and magnetotransmission in the region of technical magnetization (i.e., magnetization related to the reorientation of magnetic domains) in fields weaker than 300 Oe and that of large negative magnetoresistance (25\%) and magnetotransmission (1\%) related to the tunneling effect in magnetic fields of above $3 \mathrm{kOe}$.

\section{Acknowledgments}

The work was supported by the program UB of RAS No. 15-9-24 , by grant of the RF President for Leading scientific schools SS8003.2016.2 and by grant No. 14.250 .31 .0025 in parts, within the state assignment of FASO of Russia ("Spin" theme No. 01201463330).

\section{Appendix A. Supplementary data}

Supplementary data associated with this article can be found, in the online version, at https://doi.org/10.1016/j.jmmm.2017.10.006.

\section{References.}

[1] A. Granovsky, Y.u. Sukhorukov, E. Gan'shina, A. Telegin, Magnetorefractive effect in magnetoresistive materials, in: M. Inoue, M. Levy, A.V. Baryshev (Eds.), Magnetophotonics: From Theory to Applications, Magnetophotonics, Springer Series in Materials Science, 178, Springer Berlin Heidelberg, 2013, pp. 107-133, https://doi.org/10.1007/978-3-642-35509-7 5.

[2] A.V. Telegin, Y.u.P. Sukhorukov, N.N. Loshkareva, E.V. Mostovshchikiva, N.G. Bebenin, E.A. Gan'shina, A.B. Granovsky, Giant magnetotransmission and magnetoreflection in ferromagnetic materials, JMMM 383 (2015) 104-109, https://doi.org/10.1016/j.jmmm.2014.11.080.

[3] M. Ziese, Extrinsic magnetotransport phenomena in ferromagnetic oxides, Rep. Prog. Phys. 65 (2002) 143-249, https://doi.org/10.1088/0034-4885/65/2/202. 
[4] A.R. Kaul, O.Yu. Gorbenko, A.A. Kamenev, The role of heteroepitaxy in the development of new thin-film oxide-based functional materials, Russ. Chem. Rev. 73 (2004) 861-880, https://doi.org/10.1070/RC2004v073n09 ABEH000919.

[5] O.Y.u. Gorbenko, R.V. Demin, A.R. Kaul', L.I. Koroleva, R. Szymczak, Magnetic, electrical, and crystallographic properties of thin $\mathrm{La}_{1-\mathrm{x}} \mathrm{Sr}_{\mathrm{x}} \mathrm{MnO}_{3}$ films, Phys. Solid State 40 (1998) 263-267, https://doi.org/10.1134/1.1130289.

[6] J.S. Helman, B. Abeles, Tunneling of spin-polarized electrons and magnetoresistance in granular Ni films, Phys. Rev. Lett. 37 (1976) 14291432, https://doi.org/10.1103/PhysRevLett. 37.1429.

[7] N. Zhang, Tunnel type giant magnetoresistance in the granular perovskite $\mathrm{La}_{0.85} \mathrm{Sr}_{0.15} \mathrm{MnO}_{3}$, Phys. Rev. B 56 (1997) 8138-8142, https://doi.org/10.1103/ PhysRevB.56.8138.

[8] A.B. Granovskii, M.V. Kuz'michev, J.P. Clerc, Optical and magnetooptical properties of granular alloys with giant magnetoresistance in the IR region of the spectrum, J. Exp. Theor. Phys. 89 (1999) 955-959, https://doi.org/ $10.1134 / 1.558937$.

[9] S. Uran, M. Grimsditch, E.E. Fullerton, S.D. Bader, Infrared spectra of magnetoresistance $\mathrm{Fe} / \mathrm{Cr} / \mathrm{Fe}$ trilayers, Phys. Rev. B 57 (1998) 2705-2708, https://doi.org/10.1103/PhysRevB.57.2705.

[10] V.V. Ustinov, Y.U.P. Sukhorukov, M.A. Milyaev, A.B. Granovskii, A.N. Yurasov, E A. Gan'shina, A.V. Telegin, J. Exp. Theor. Phys. 108 (2009) 260-266, https://doi. org/10.1134/S1063776109020083.

[11] Y.u.P. Sukhorukov, A.V. Telegin, E.A. Gan'shina, N.N. Loshkareva, A.R. Kaul', O.Y. u. Gorbenko, E.V. Mostovshchikova, O.V. Mel'nikov, A.N. Vinogradov, Tunneling of spin-polarized charge carriers in $\mathrm{La}_{0.8} \mathrm{Ag}_{0.1} \mathrm{MnO}_{3+\delta}$ film with variant structure: magnetotransport and magnetooptical data, Tech. Phys. Lett. 31 (2005) 484-487, https://doi.org/10.1134/1.1969772.

[12] Yu.P. Sukhorukov, A.V. Telegin, A.B. Granovskii, E.A. Gan'shina, S.V. Naumov, N. V. Kostromitina, L.V. Elokhina, J. Gonzalez, Magnetorefractive effect in $\mathrm{La}_{0.7} \mathrm{Ca}_{0.3} \mathrm{MnO}_{3}$ in the infrared spectral range, J. Exp. Theor. Phys. 138 (2010) 355-362, https://doi.org/10.1134/S1063776110090037.

[13] C. Roy, R.C. Budhani, Raman, infrared and x-ray diffraction study of phase stability in $\mathrm{La}_{1-\mathrm{x}} \mathrm{Ba}_{\mathrm{x}} \mathrm{MnO}_{3}$ doped manganites, J. Appl. Phys. 85 (1999) 3124 3131, https://doi.org/10.1063/1.369651.
[14] N.G. Bebenin, N.N. Loshkareva, A.A. Makhnev, E.V. Mostovshchikova, L.V. Nomerovannaya, E.A. Gan'shina, A.N. Vinogradov, M.Ya. Mukovskii, Optical and magnetooptical properties of ferromagnetic $\mathrm{La}_{1-\mathrm{x}} \mathrm{Ba}_{\mathrm{x}} \mathrm{MnO}_{3}$ single crystals, J. Phys. Condens. Matter. 22 (2010) 096003, https://doi.org/10.1088/09538984/22/9/096003.

[15] E. Ganshina, N. Loshkareva, Y.u. Sukhorukov, E. Mostovshchikova, A. Vinogradov, L. Nomerovannaya, Optical and magneto-optical spectroscopy of manganites, JMMM 300 (2006) 62-66, https://doi.org/10.1016/j. jmmm.2005.10.033.

[16] A.S. Moskvin, I.L. Avvakumov, Doped manganites beyond conventional doubleexchange model, Phys. B 322 (2002) 371-389, https://doi.org/10.1016/S09214526(02)01275-9.

[17] A.S. Moskvin, Disproportionation and electronic phase separation in parent manganite $\mathrm{LaMnO}_{3}$, Phys. Rev. B 79 (2009) 115102, https://doi.org/10.1103/ PhysRevB.79.115102.

[18] A.N. Yurasov, Magnetorefractive effect as contactless method for study of functional materials, Materialovedenie 6 (2014) 32-38 (in Russian).

[19] S. Mitani, S. Takanashi, K. Takanashi, K. Yakushiji, S. Maekawa, H. Fujimori, Enhanced magnetoresistance in insulating granular systems: evidence for higher-order tunneling, Phys. Rev. Lett. 81 (1998) 2799-2802, https://doi.org/ 10.1103/PhysRevLett. 81.2799.

[20] H.Y. Hwang, S.-W. Cheong, N.P. Ong, B. Batlogg, Spin-polarized intergrain tunneling in $\mathrm{La}_{2 / 3} \mathrm{Sr}_{1 / 3} \mathrm{MnO}_{3}$, Phys. Rev. Lett. 77 (1996) 2041-2044, https://doi. org/10.1103/PhysRevLett. 77.2041.

[21] B. Vengalis, G. Grigaliūnaite-Vonsevičiene, A. Maneikis, J. Klimantavičius, R. Juškenas, K. Mažeika, Low field magnetoresistance and swiching behavior of polycrystalline $\mathrm{La}_{0.66} \mathrm{Sr}_{0.34} \mathrm{MnO}_{3}$, YSZ(001) films with columnar grain structure, Thin Solid Films 625 (2017) 42-48, https://doi.org/10.1016/j. tsf.2017.01.040.

[22] R. Shreekala, M. Rajeswari, K. Ghosh, A. Goyal, J.Y. Gu, C. Kwon, Z. Trajanovic, T. Boettcher, R.L. Greene, R. Ramesh, T. Venkatesan, Effect of crystallinity on the magnetoresistance in perovskite manganese oxide thin films, Appl. Phys. Lett. 71 (1997) 282-284, https://doi.org/10.1063/1.119520. 